Check for updates

Cite this: RSC Adv., 2019, 9, 9323

Received 18th January 2019

Accepted 18th March 2019

DOI: $10.1039 / \mathrm{c} 9 \mathrm{ra00458k}$

rsc.li/rsc-advances

\section{Discovery of human TyrRS inhibitors by structure- based virtual screening, structural optimization, and bioassays $\uparrow$}

\author{
Shenzhen Huang, (ID $\ddagger^{a}$ Xiang Wang, $t^{\text {bc }}$ Guifeng Lin, ${ }^{a}$ Jie Cheng, ${ }^{a}$ Xiuli Chen, ${ }^{a}$ \\ Weining Sun, ${ }^{d}$ Rong Xiang, ${ }^{b}$ Yamei Yu, ${ }^{a}$ Linli Lid and Shengyong Yang iD *a
}

\section{Introduction}

Resveratrol (3,4,5-trihydroxystilbene, RSV), a natural product, is an important component of red wine. RSV has long been thought to impart benefits in lifespan expectancy, ${ }^{\mathbf{1 , 2}}$ cancer chemopreventive activity, ${ }^{3}$ cardioprotective, ${ }^{4}$ and neuroprotective effects, ${ }^{5}$ by launching a stress response. ${ }^{6,7}$ However, because it has low activity and the target of its action is not clear ${ }^{8}$ RSV is only marketed as a health care product. Human tRNA synthetase is a potent PARP1-activating effector target for RSV.

Recently, Sajish et al. demonstrated that RSV could interact with human tyrosyl transfer-RNA synthetase (TyrRS) and hence play its beneficial role. ${ }^{7}$ TyrRS was originally known for its essential aminoacylation function in protein synthesis. Recent studies have found a new function for TyrRS in DNA damage protection. ${ }^{7,9,10}$ External stimuli, such as oxidative stress, can induce TyrRS to rapidly translocate from the cytosol to the

${ }^{a}$ State Key Laboratory of Biotherapy and Cancer Center, West China Hospital, Sichuan University/Collaborative Innovation Center of Biotherapy, Chengdu, Sichuan 610041, China.E-mail: yangsy@scu.edu.cn

${ }^{b}$ Department of Clinical Medicine, School of Medicine, Nankai University, Tianjin 300071, China

'Department of Chemistry, Fudan University, Shanghai 200433, China

${ }^{d}$ Key Laboratory of Drug Targeting and Drug Delivery System of Ministry of Education, West China School of Pharmacy, Sichuan University, Chengdu, Sichuan 610041, China $\dagger$ Electronic supplementary information (ESI) available: General procedure for the synthesis of compounds (1-31), Fig S1-S7, and Table S1. See DOI: 10.1039/c9ra00458k

\$ These authors contributed equally to this work. nucleus. The nuclear-localized TyrRS activates transcription factor E2F1 to upregulate the expression of DNA damage repair genes such as BRCA1 and RAD51. ${ }^{9}$ In particular, RSV can also trigger the translocation of TyrRS to the nucleus by directly binding to the active site of TyrRS, leading to the activation of $\mathrm{NAD}^{+}$-dependent auto-poly-ADP ribosylation of poly(ADP ribose) polymerase 1 (PARP-1). PARP-1 is a major stress response and DNA repair factor that has a considerable impact on longevity. ${ }^{11}$ PARP-1 activated by TyrRS leads to activation of a series of protective genes, including the tumor suppressor gene p53, FOXO3A, or SIRT6. ${ }^{7}$ Therefore, TyrRS is an attractive target for developing therapeutics for diseases of the nervous system diseases and for DNA damage protection.

To date, RSV is the only small molecule compound with an inhibition constant $\left(K_{\mathrm{i}}\right)$ value in the micromolar range that has been identified as an inhibitor of TyrRS. ${ }^{7}$ However, RSV has also been identified as a modulator against many targets. ${ }^{\mathbf{1 2 - 1 5}}$ Therefore, discovery of novel potent and selective TyrRS inhibitors, with scaffolds different from RSV, is desirable.

Here, we report the discovery of a new series of TyrRS inhibitors containing the scaffold 5,7-dihydroxy-2-phenyl-6,8bis(phenylthio)- $4 H$-chromen-4-one. These compounds were identified by a high throughput virtual screening and subsequent structure-activity relationship (SAR) analysis. The most active compound 5,7-dihydroxy-6,8-bis((3-hydroxyphenyl)thio)2-phenyl- $4 H$-chromen-4-one (11) was further investigated in binding mode analysis with TyrRS and bio-functional studies. $^{7,9,10,16,17}$ Compound $\mathbf{1 1}$ is a promising lead compound for the further development of drugs for DNA damage related diseases. 


\section{Results and discussion}

\section{Structure-based virtual screening of novel hit compounds against TyrRS}

The virtual screening process is schematically shown in Fig. 1A. The chemical databases included the commercial chemical library Specs and an in-house database. In the first step, compounds were filtered by Lipinski's rules and for pan-assay interference compounds (PAINS) in Pipeline Pilot 8.5, which is used for filtering pan-assay interference compounds ${ }^{\mathbf{1 8 , 1 9}}$ and has been used with great success in drug discovery. ${ }^{\mathbf{1 8 , 2 0}}$ Then molecular docking was carried out with the remaining compounds. Here, the GOLD program was used because it provides good docking performance. ${ }^{21}$ All the calculations were carried out on the platform of Accelrys Discovery Studio 3.1 (Accelrys Inc., San Diego, CA, USA). The crystal structure of TyrRS in complex with RSV (PDB entry 4Q93) was used for the docking studies, ${ }^{7}$ and RSV was used as a reference molecule with a root-mean-square deviation (RMSD) of $0.57 \AA$ (Fig. S2 $\dagger$ ). Next, the consensus scoring strategy was used to score the compounds that may target TyrRS. Here, the scoring functions, GoldScore incorporated in the GOLD program and ID-Score developed by our laboratory, ${ }^{22}$ were selected for the consensus scoring strategy because they have provide good performance for scoring. ${ }^{18,22}$ Finally, 30 compounds were selected from the top ranked compounds by the consensus scoring and visual inspection.

\section{TyrRS enzyme inhibition assays}

The 30 in silico-screened compounds were tested for inhibitory activity against TyrRS using the ATP-pyrophosphate exchange assay (Fig. 1B). From the enzyme inhibition assay, the hit compounds AK-087/41343686, AH-357/03514064, and SKLB2002 were identified, they showed an enzyme activity < $60 \%$ against TyrRS at the concentration of $100 \mu \mathrm{M}$ and inhibited TyrRS activity in biochemical assays in a dose-dependent

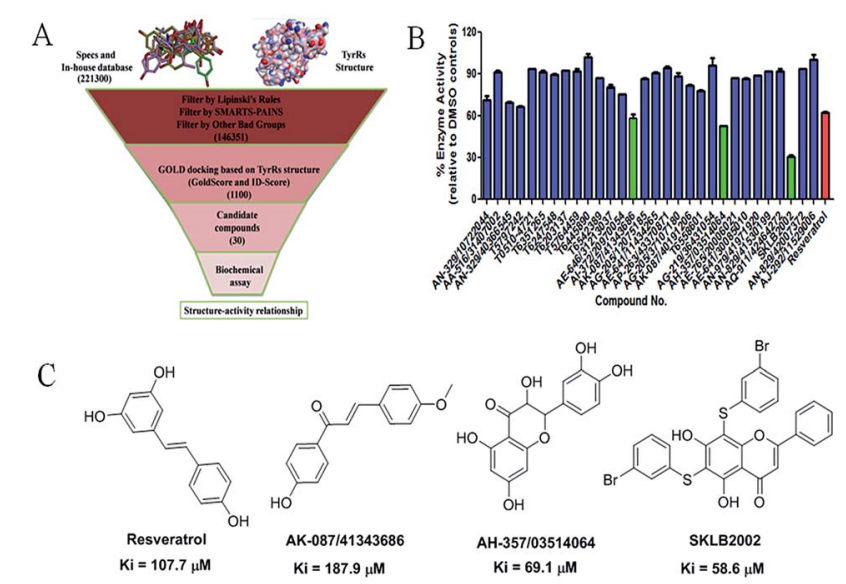

Fig. 1 (A) Schematic diagram of the underlying workflow of the TyrRS drug discovery. (B) Enzyme activity of the 30 candidate molecules based on virtual screening at $100 \mu \mathrm{M}$. The red columnar bar represents the activity of the reference compound RSV. (C) The structures of the hit compounds and RSV. manner with $K_{\mathrm{i}}$ values of $187.9,69.1$, and $58.6 \mu \mathrm{M}$, respectively. The positive control compound RSV showed weak potency $\left(K_{\mathrm{i}}=107.7 \mu \mathrm{M}\right)$ (Fig. 1C and S1†). Because the hit compounds AH-357/03514064 and SKLB2002 have similar scaffolds, and compound SKLB2002 was the most active compound and contained the new scaffold 5,7-dihydroxy-2phenyl- $4 H$-chromen-4-one, further structural optimization and SAR analysis were carried out on this compound.

\section{Chemistry}

All of the target compounds were prepared from the commercially available 5,7-dihydroxy-2-phenyl-4H-chromen-4-one. The general synthetic routes for compounds 1 (SKLB2002), 2-12, and 14-31 shown in Scheme 1. In route 1, intermediate 1a was prepared by a similar synthetic method as described in the literature. ${ }^{23}$ Target compounds 1-12 were then obtained by a conventional coupling reaction of various benzenethiols and intermediate $1 \mathrm{a}$ in DMF in yields of $40-70 \%$. In route 2 , intermediate 13a was obtained by a displacement reaction with iodomethane and commercially available 5,7-dihydroxy-2phenyl- $4 H$-chromen-4-one. Intermediate $\mathbf{1 3 b}$ was then obtained by a classic electrophilic substitution reaction between NIS and the previously synthesized intermediate 13a in quantitative yield. Target compounds 14-22 were synthesized from various benzenethiols and intermediate 13b in DMF. Finally, the target compounds 23-31 were obtained by demethylation of compounds 14-22 with boron tribromide. ${ }^{24}$

\section{SAR analysis and optimization of hit compounds}

The inhibitory effects of the synthesized compounds on human TyrRS are displayed in Tables 1 and 2 . As can be seen from Table 1 , the introduction of methyl, methoxyl, and tert-butyl groups, that is compounds $3, \mathbf{6}, \mathbf{8}$, and $\mathbf{9}$, which substantially diminished activity. The series of derivatives $(\mathbf{1}, \mathbf{2}, \mathbf{5}, \mathbf{7}, \mathbf{1 1}$, and 12) revealed that the presence of halogen or hydroxyl groups in the

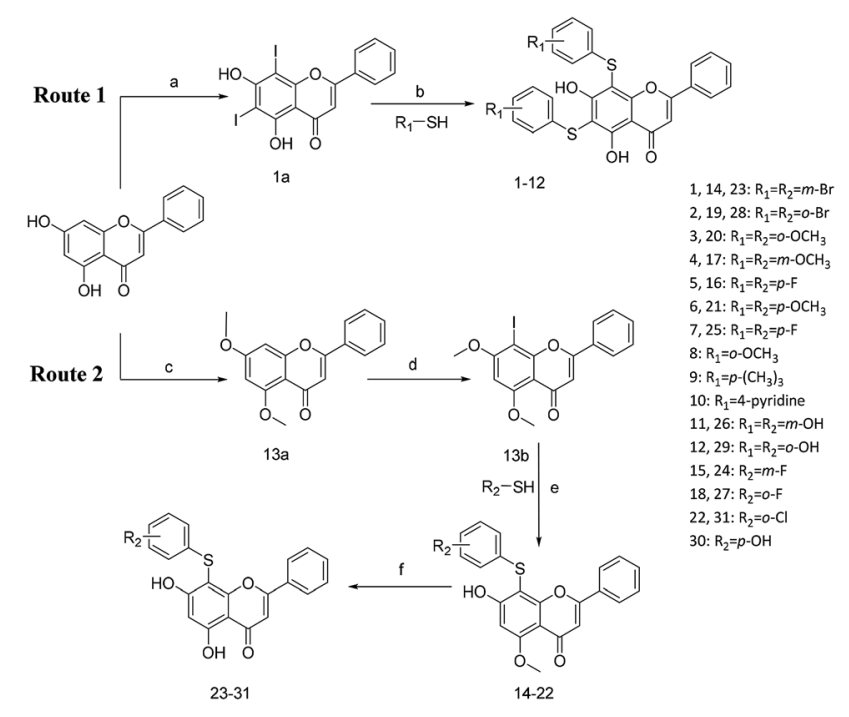

Scheme 1 Reagents and conditions: (a) $\mathrm{HNO}_{3}, \mathrm{I}_{2}, \mathrm{CH}_{3} \mathrm{COOH}$, RT. (b and e) $\mathrm{Pd}_{2}(\mathrm{dba})_{3}, \mathrm{X}$-phos, $\mathrm{Cs}_{2} \mathrm{CO}_{3}, \mathrm{DMF}, \mathrm{N}_{2}, 110{ }^{\circ} \mathrm{C}$. (c) $\mathrm{CH}_{3} \mathrm{I}, \mathrm{K}_{2} \mathrm{CO}_{3}$, DCM, RT. (d) NIS, $\mathrm{CF}_{3} \mathrm{COOH}, 80^{\circ} \mathrm{C}$. (f) $\mathrm{BBr}_{3}, \mathrm{DCM}, \mathrm{RT}$. 
Table 1 Chemical structures and bioactivities of SKLB2002 derivatives with $R_{1}$ varied

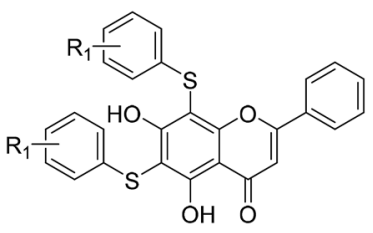

\begin{tabular}{lll}
\hline Cpd & $\mathrm{R}_{1}$ & Enzyme activity@100 $\mu \mathrm{M}(\%)$ \\
\hline $\mathbf{1}$ & $m-\mathrm{Br}$ & 45.63 \\
$\mathbf{2}$ & $o-\mathrm{Br}$ & 31.76 \\
$\mathbf{3}$ & $o-\mathrm{OCH}_{3}$ & 88.86 \\
$\mathbf{4}$ & $m-\mathrm{OCH}_{3}$ & 53.87 \\
$\mathbf{5}$ & $p-\mathrm{F}$ & 47.76 \\
$\mathbf{6}$ & $p-\mathrm{CH}_{3}$ & 76.17 \\
$\mathbf{7}$ & $m-\mathrm{F}$ & 19.24 \\
$\mathbf{8}$ & $o-\mathrm{CH}_{3}$ & 75.93 \\
$\mathbf{9}$ & $p-\left(\mathrm{CH}_{3}\right)_{3}$ & 85.29 \\
$\mathbf{1 0}$ & $4-\mathrm{Pyridine}$ & 24.48 \\
$\mathbf{1 1}$ & $m-\mathrm{OH}$ & 4.57 \\
$\mathbf{1 2}$ & $o-\mathrm{OH}$ & 29.92
\end{tabular}

${ }^{a}$ The pyridine ring was use to replace the benzenoid structure.

skeletal structure of these molecules was desirable; compound $\mathbf{7}$ and, in particular, compound 11, displayed an inhibition rate larger than $80 \%$ at a concentration of $100 \mu \mathrm{M}$. Compounds 7 and 11 strongly inhibited TyrRS with $K_{\mathrm{i}}$ values of 10.6 and 8.8 $\mu \mathrm{M}$, respectively, which were considerably higher than that shown by the positive control RSV $\left(K_{\mathrm{i}}=107.7 \mu \mathrm{M}\right.$, Fig. 2 and $\mathrm{S} 1 \dagger)$.

As can be seen from Table 2 , the $R_{2}$ and $R_{3}$ groups were also optimized. However, deletion of the benzenethiol-substituted unit at the 18-position of in the skeletal structure of these molecules was not ideal. Compounds 14-31 showed weaker potency compared with the benzenethiol-substituted compounds. Compound $11, K_{\mathrm{i}}=8.8 \mu \mathrm{M}$, was the most active compound and was the most promising inhibitor in the present series of derivatives.

\section{Differential scanning fluorimetry (DSF) and differential scanning calorimetry (DSC) assays}

DSF is a good platform for screening protein stability conditions and has the advantages of being fast and inexpensive. Because of the small amount and low concentration of protein required, DSF can be used to identify low-molecular-weight ligands. A DSF assay was employed to understand the relationship between TyrRS inhibitor binding and protein construct stability $^{25,26}$ (Fig. 3A and B). The impact of the binding of RSV and compound $\mathbf{1 1}$ on the TyrRS was explored by determining the changes in the melting temperature $\left(T_{\mathrm{m}}\right)$ of the protein in the absence and the presence of ligand. The shift in the $T_{\mathrm{m}}$ as defined by the relative midpoint of the denaturation profile assay indicated that the thermal stability was dependent on the ligand concentration. As shown in Fig. 3A, the presence of compound $\mathbf{1 1}$ increased the $T_{\mathrm{m}}$ value of the native TyrRS protein, showing that this class of inhibitor retained the ability to bind and stabilize the protein construct. Similarly, a significant increase in the $T_{\mathrm{m}}$ value was also observed for RSV, a specific binding ligand for this protein (Fig. 3B).

DSC, a thermal analysis method, has become an efficient experimental technique for determination of the thermodynamic properties of biological macromolecules such as proteins. ${ }^{27-29}$ A DSC assay was also performed to determine the $T_{\mathrm{m}}$ values for TyrRS. In the DSC assay, the $T_{\mathrm{m}}$ values of the TyrRS protein in the absence and presence of ligands were determined by the midpoint of the rise in each curve ${ }^{\mathbf{3 0 , 3 1}}$ (Fig. 3C and D). The results obtained were comparable with the DSF method and showed the same trends. As shown in Fig. 3C and D, the presence of RSV or compound $\mathbf{1 1}$ shifted the native TyrRS protein $T_{\mathrm{m}}$ value by more than 1.84 or $1.30{ }^{\circ} \mathrm{C}$ higher, respectively.

Table 2 Chemical structures and bioactivities of SKLB2002 derivatives with $R_{2}$ and $R_{3}$ varied

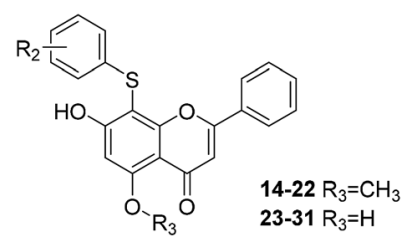

\begin{tabular}{|c|c|c|c|c|c|}
\hline Cpd & $\mathrm{R}_{2}$ & Enzyme activity@100 $\mu \mathrm{M}(\%)$ & Cpd & $\mathrm{R}_{2}$ & Enzyme activity@100 $\mu \mathrm{M}(\%)$ \\
\hline 14 & $m-\mathrm{Br}$ & 86.42 & 23 & $m-\mathrm{Br}$ & 91.4 \\
\hline 15 & $m-\mathrm{F}$ & 68.51 & 24 & $m-\mathrm{F}$ & 75.12 \\
\hline 17 & $m-\mathrm{OCH}_{3}$ & 71.84 & 26 & $m-\mathrm{OH}$ & 93.16 \\
\hline 18 & $o-\mathrm{F}$ & 73.76 & 27 & $o-\mathrm{F}$ & 87.04 \\
\hline 19 & $o-\mathrm{Br}$ & 69.55 & 28 & $o-\mathrm{Br}$ & 72.44 \\
\hline 22 & $m-\mathrm{Cl}$ & 77.86 & 31 & $m-\mathrm{Cl}$ & 87.07 \\
\hline
\end{tabular}


A

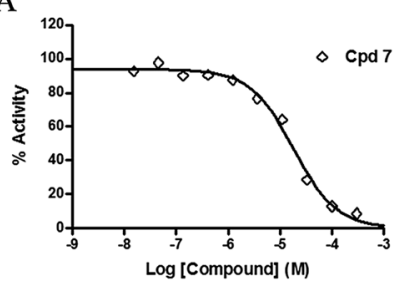

B

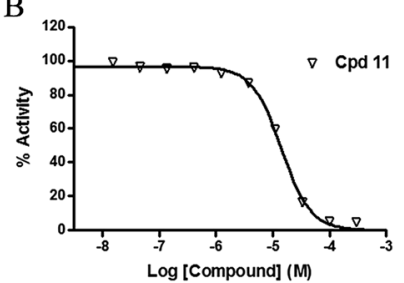

Fig. 2 Enzyme activity data for TyrRS with compounds 7 and 11. (A and B) Determination of the $K_{\mathrm{i}}$ values of compounds 7 and 11 , respectively.

A
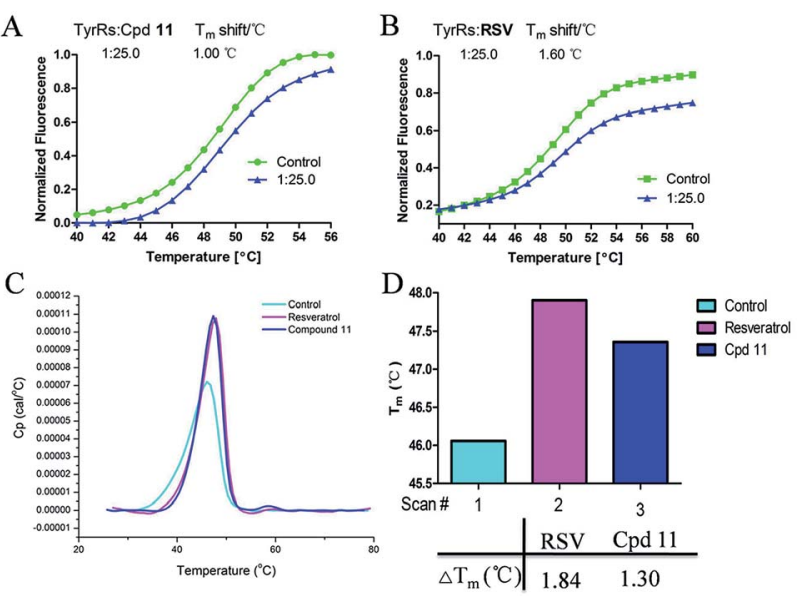

Fig. 3 DSF and DSC were used to test the stabilization of TyrRS by RSV and compound 11. (A) The $T_{m}$ value of compound 11. (B) The $T_{m}$ value of RSV. Also shown are graphs of the unfolding transition of $2 \mu \mathrm{M}$ TyrRS in the presence of $50 \mu \mathrm{M}$ RSV or compound 11, respectively. (C and D) Representative DSC melting profiles for TyrRS with RSV or compound 11; $\Delta T_{\mathrm{m}}$ of RSV and compound 11 obtained from DSC.

\section{Binding-mode analysis}

Fig. 4 shows a possible binding mode of compound 11 with TyrRS. For comparison, RSV was also shown in the active site of TyrRS (PDB entry 4Q93). Obviously compound 11 occupies the same binding site but a larger area than RSV does. The $3-\left(\lambda^{1}-\right.$

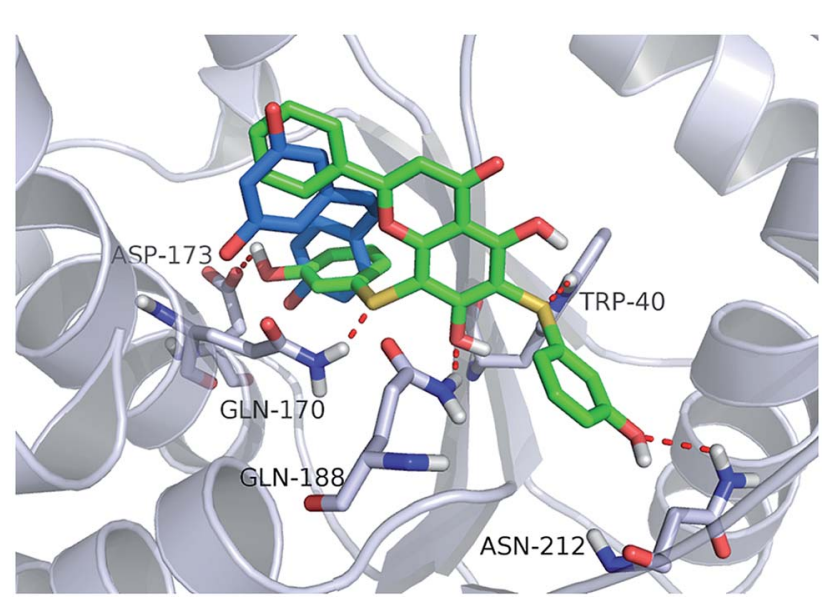

Fig. 4 Binding modes of compound 11 and RSV with TyrRS (PDB code 4 Q93). Compound 11 is shown as green sticks, and RSV is displayed as marine sticks. sulfanyl)phenol group at the 6, 8-position of 5,7-dihydroxy-2phenyl- $4 H$-chromen-4-one is orientated with the $\mathrm{H}$ atom of the $\mathrm{OH}$ group and the $\mathrm{S}$ atom hydrogen-bonded to the Asp173 or Trp40 residues in the active site of TyrRS. The $\mathrm{O}$ atom in the 7hydroxy branch of compound $\mathbf{1 1}$ formed another hydrogenbonding interaction with the Gln170 residue. Compound 11 also formed hydrophobic interactions with residues Tyr39, Tyr52, Leu72, and Phe183 in the active site of TyrRS.

\section{Protection against DNA damage}

DNA damage can be induced by various stimuli, including UV radiation from the environment, oxygen produced by endogenous reactive oxygen species, and chemicals for disease treatment. DNA damage is considered a major source of gene mutations and a cause of many diseases, for example, neurodegenerative diseases, cardiovascular disease, immune dysfunction, and cancer. $^{32,33}$ Even though there is a DNA damage response in cells to fight against DNA damage caused by endogenous or environmental stimulus, it is not sufficient for cells to eliminate all DNA damage. Thus, chemicals that can help cells fight against DNA damage are desirable. Serum starvation (SS, oxidative stress) and RSV have been shown to affect TyrRS nuclear localization. ${ }^{7,9}$ This observation prompted us to investigate whether compound $\mathbf{1 1}$ can regulate TyrRS nuclear localization in HeLa cells. By immunoblotting of the cytoplasmic and nuclear fractions from cells, we found that treatment with compound $\mathbf{1 1}$ dramatically promoted the nuclear localization of TyrRS in a concentration-dependent manner in HeLa cells, without the influence of TyrRS in the cytoplasm (Fig. 5).

Having demonstrated that nuclear localization of TyrRS is promoted by compound 11, we investigated the potential for TyrRS inhibitors to protect cells from DNA damage. We pretreated HeLa cells with compound 11 for $24 \mathrm{~h}$ before inducing DNA damage in cells by treatment with $30 \mu \mathrm{M}$ cisplatin for $24 \mathrm{~h}$. To visualize the DNA damage level in cells, phosphorylated histone H2A.X at S139 ( $\gamma$-H2A.X), used as a biomarker for DNA double-strand breaks, was detected by immunoblotting. Without cisplatin treatment, the level of $\gamma$-H2A.X was below the detection limit (Fig. 6). However, with cisplatin treatment, $\gamma$ H2A.X could be detected. We found that treatment with compound 11 dramatically decreased the level of $\gamma-\mathrm{H} 2 \mathrm{~A} . \mathrm{X}$ in HeLa cells, suggesting that nuclear TyrRS had a protective effect on DNA damage. Compound 7 had a similar effect as shown in Fig. S7.†

\section{Experimental}

\section{Ligand preparation}

Compound libraries, including In-house and Specs databases, were used for virtual screening in this investigation. The total number of compounds is approximately 220000 . All the compounds were used for docking, which were prepared with 'Prepare Ligands' module in Discovery Studio (DS) 3.1 (Accelrys Inc., San Diego, CA, USA). Parameter values for "Change Ionization, Generate Tautomers, and Generate Isomers" were set to 

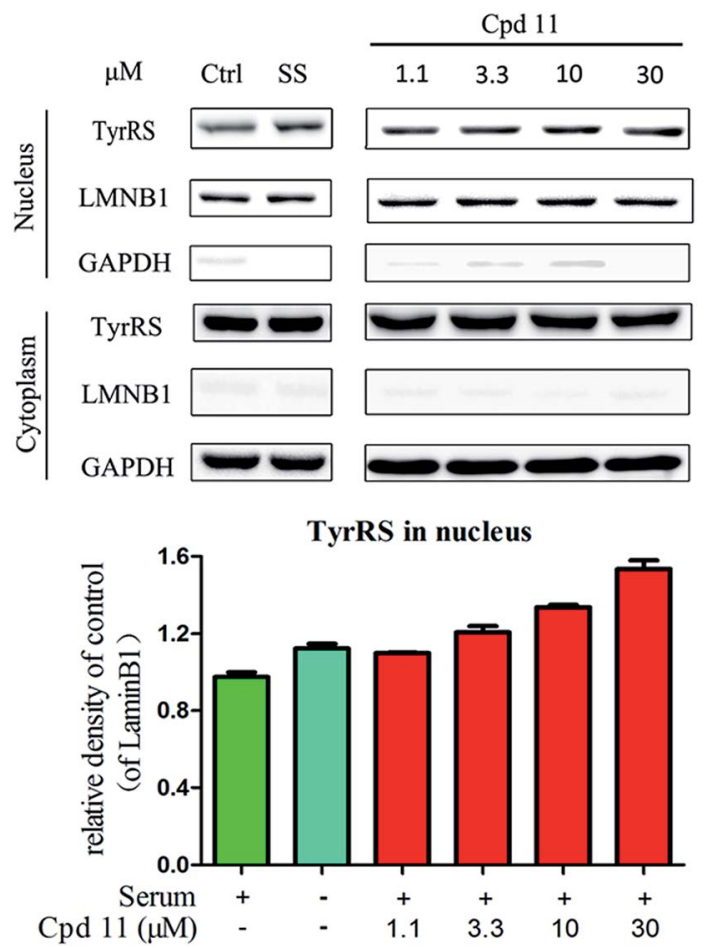

Fig. 5 Compound 11 promoted TyrRS nuclear localization in HeLa cells. Hela cells were treated with compound $11(1.1,3.3,10$, or $30 \mu \mathrm{M})$ or DMSO for $8 \mathrm{~h}$. Cells cultured in serum-free media for $8 \mathrm{~h}$ were under serum starvation. The cytoplasmic and nuclear fractions from cells were subjected to immunoblot assays. Quantification of immunoblots is shown below.

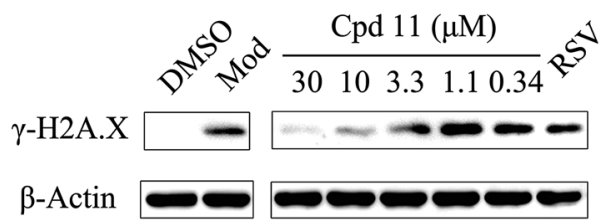

Fig. 6 Nuclear TyrRS promoted by compound 11 protected cells from DNA damage in HeLa cells. HeLa cells were pre-treated with compound $11(30,10,3.3,1.1$, or $0.37 \mu \mathrm{M})$, RSV $(1 \mu \mathrm{M})$, or DMSO for $24 \mathrm{~h}$. To induce DNA damage, cells were treated with cisplatin $(30 \mu \mathrm{M})$ or DMSO in the presence of the test compounds or DMSO for $24 \mathrm{~h}$. Then, whole cell lysates were subjected to immunoblot assays.

false. Other parameters were set to their default values. The compounds were retrieved according to previous reported..$^{\mathbf{1 8 , 3 4 , 3 5}}$ Selected compounds were purchased in milligram quantities from chemical vendors or synthesis by our laboratory. Purity of compounds was $\geq 95 \%$, as declared by the chemical vendors.

\section{Structure-based virtual screening of novel hit compounds against TyrRS}

In this investigation, the docking method GOLD was involved in the platform of DS 3.1. Before molecular docking, the receptor protein was prepared by the DS 3.1 software package with standard preparation procedures (protein preparation protocol), which include removing water molecules, adding hydrogen atoms to the protein, and assigning force field (here the CHARMm forcefield was adopted). For GOLD, the "predefined generic algorithm (GA)" setting of 'automatic' was employed and others were set to default.

\section{General procedure for the synthesis of compounds 1-31}

All commercially available starting materials, solvents, and reagents were obtained from commercial suppliers and used without further purification unless otherwise indicated. Reactions were monitored by thin layer chromatography (TLC) on Merck silica gel 60 F-254 thin layer plates. ${ }^{1} \mathrm{H}$ NMR spectra was recorded on a Bruker AV-400 spectrometer at $400 \mathrm{MHz}$. Spin multiplicities are described as s (singlet), br. s (broad singlet), $\mathrm{t}$ (triplet), q (quartet), and $\mathrm{m}$ (multiplet). Chemical shifts $(\delta)$ are listed in parts per million ( $\mathrm{ppm}$ ) relative to tetramethylsilane (TMS) as an internal standard. Mass spectral (MS) data were acquired on a Waters Q-TOF Premier mass spectrometer (Micromass, Manchester, U.K.). The ${ }^{1} \mathrm{H}$ NMR, ${ }^{13} \mathrm{C}$ NMR, and MS of compounds 1-31 are listed in the ESI. $\dagger$

\section{Purification of human TyrRS protein}

Human TyrRS was purified as previously described. ${ }^{7,36,37}$ The plasmid of mini-TyrRS (amino acids 1-341) as a gift from Paul Schimmel. Briefly, this plasmid was transformed into E. coli Arctic Express (DE3) cells. The cells were cultured at $37{ }^{\circ} \mathrm{C}$ in fresh LB liquid medium ( $5 \mathrm{~g}$ of $\mathrm{NaCl}, 10 \mathrm{~g}$ of bactotrypton, and $10 \mathrm{~g}$ of yeast extract per litre). Isopropyl- $\beta$-D-1-thiogalactopyranoside (IPTG, $0.5 \mathrm{mM}$ ) was used to induce expression when attenuance $D_{600 \mathrm{~nm}}$ was 1 , and the culture was grown for $20 \mathrm{~h}$ at $16{ }^{\circ} \mathrm{C}$. Cells were collected by centrifugation at $4000 \mathrm{rpm} \mathrm{min}{ }^{-1}$ for $15 \mathrm{~min}$ at $4{ }^{\circ} \mathrm{C}$ and then re-suspended in lysis buffer $(50 \mathrm{mM}$ Hepes-Na, pH 7.5, 5 mM imidazole pH 7.5, $400 \mathrm{mM} \mathrm{NaCl}, 1 \times$ PMSF, and $2 \mathrm{mM} \mathrm{BME).} \mathrm{Cells} \mathrm{were} \mathrm{lysed} \mathrm{using} \mathrm{a} \mathrm{cell} \mathrm{disrupter.}$ After centrifugation at $14000 \mathrm{rpm} \mathrm{min}{ }^{-1}$ for $25 \mathrm{~min}$ at $4{ }^{\circ} \mathrm{C}$, the supernatant was loaded onto a nickel-affinity chromatography column (GE-Healthcare) pre-equilibrated with lysis buffer. The protein was eluted with a linear gradient of imidazole (0-300 $\mathrm{mM})$. The desired fractions were pooled, concentrated and subjected to gel-filtration (Superdex 200; GE-Healthcare) chromatography and the protein peak corresponding to homogenous protein in buffer (20 mM Hepes-Na, pH 7.5, $50 \mathrm{mM} \mathrm{NaCl}$, and $2 \mathrm{mM}$ BME) was collected (see Fig. S3†). The quality of the protein purification was validated by SDS-PAGE analysis (see Fig. S4†).

\section{In vitro enzymatic inhibition assays}

In vitro enzymatic inhibition assays were performed using an ATP-pyrophosphate exchange assay as previously described ${ }^{7}$ and the results provided by Reaction Biology Corp, Malvern, United States. Briefly, tyrosyl adenylate synthesis was measured using the tyrosine-dependent ATP-pyrophosphate (PPi) exchange assay. A mixture containing $100 \mathrm{mM}$ HEPES-Na ( $\mathrm{pH}$ 7.5), $20 \mathrm{mM} \mathrm{KCl,} 2 \mathrm{mM}$ ATP, $1 \mathrm{mM} \mathrm{NaPPi}, 2 \mathrm{mM}$ DTT, $100 \mu \mathrm{M} \mathrm{L}^{-}$ tyrosine (T3754-50G, Sigma), 0.02\% Brij35 (w/v), $10 \mathrm{mM} \mathrm{MgCl}$, and approximately $0.01 \mathrm{mCi} \mathrm{ml}^{-1} \mathrm{Na}[32 \mathrm{P}] \mathrm{PPi}$ (NEX019001MC, PerkinElmer) was added to $12.5 \mathrm{nM}$ purified TyrRS, pre- 
incubated with $0-300 \mu \mathrm{M}$ of test compounds or $0-1000 \mu \mathrm{M}$ of positive control RSV (ref. 7) at $4{ }^{\circ} \mathrm{C}$ for $30 \mathrm{~min}$. The ATP-PPi exchange reaction mixture was incubated at room temperature for $2 \mathrm{~h}$, and aliquots were removed at specified time intervals and quenched in a mixture containing $40 \mathrm{mM} \mathrm{NaPPi}, 1.4 \%(\mathrm{w} / \mathrm{v})$ $\mathrm{HClO}_{4}, 0.4 \%(\mathrm{w} / \mathrm{v}) \mathrm{HCl}$, and $8 \%(\mathrm{w} / \mathrm{v})$ of activated charcoal. After thorough mixing, the charcoal was filtered and washed with a solution of $7 \% \mathrm{HClO}_{4}$ (w/v) and $200 \mathrm{mM}$ NaPPi using Spin-X Centrifuge Filters (Corning) containing $0.45 \mu \mathrm{m}$ pore-size cellulose acetate filters. After drying, the charcoal was punched into scintillation vials and the radioactivity of the ATP bound to the charcoal mixture was measured by scintillation counting. The results of the L-tyrosine $K_{\mathrm{m}}$ values and enzyme titration can be found in Fig. S5 and $\mathrm{S} 6 . \dagger$ The $K_{\mathrm{i}}$ value was calculated by the equation $K_{\mathrm{i}}=\mathrm{IC}_{50} /\left(1+[\mathrm{S}] / K_{\mathrm{m}}\right)$.

\section{Differential scanning calorimetry (DSC) assay}

Human TyrRS ( $1 \mathrm{mg} \mathrm{mL}^{-1}$ ) was diluted in buffer (20 mM Hepes$\mathrm{Na}, \mathrm{pH} 7.4$, and $400 \mathrm{mM} \mathrm{NaCl}$ ) and melted using a Microcal VP Capillary DSC (GE Healthcare Life Sciences). Data were collected at $20-110{ }^{\circ} \mathrm{C}$, with a $200{ }^{\circ} \mathrm{C} \mathrm{h}^{-1}$ scan rate and $10 \mathrm{~s}$ filtering period. Data were analyzed in origin software. TyrRS was incubated with $200 \mu \mathrm{M}$ RSV or compound $\mathbf{1 1}$ for at least $0.5 \mathrm{~h}$ before melting.

\section{Differential scanning fluorimetry (DSF) assay}

DSF experiments were performed on a RT-PCR detection system (BIO-RAD CFX96) according to the known protocol. ${ }^{25}$ SYPRO orange $(5000 \times$, Invitrogen) was monitored using the filters of FRET at the wavelength of $492 \mathrm{~nm}$ for excitation and of ROX at the wavelength of $610 \mathrm{~nm}$ for emission. Each reaction solution containing $2 \mu \mathrm{M}$ protein in buffer (20 mM Hepes- $\mathrm{Na}, \mathrm{pH} 7.4$, and $100 \mathrm{mM} \mathrm{NaCl}), 5 \times$ SYPRO orange, and compound $(10 \mu \mathrm{L})$ was heated from 25 to $95{ }^{\circ} \mathrm{C}$. Fluorescence intensities were recorded every $1{ }^{\circ} \mathrm{C} \mathrm{min}^{-1}$ and plotted as a function of temperature. The inflection point of the transition curve $\left(T_{\mathrm{m}}\right)$ was calculated by fitting the Boltzmann equation to the sigmoidal curve in GraphPad Prism 5.0. Each condition was tested in triplicate.

\section{Cell cultures}

HeLa cells were maintained in Dulbecco's modified Eagle's medium which was supplemented with $10 \%(\mathrm{v} / \mathrm{v})$ fetal bovine serum. To mimic oxidative stress in cells, serum starvation under which condition cells were cultured in serum-free media was used. Cells were grown at $37{ }^{\circ} \mathrm{C}$ and $5 \% \mathrm{CO}_{2}$ in a humidified incubator.

\section{Detection of DNA damage in cells}

To determine the protection of TyrRS inhibitors against DNA damage, HeLa cells pre-seeded in 6-well plate were pre-treated with compound $7(30,10,3.3,1.1$, or $0.37 \mu \mathrm{M})$, compound 11 $(30,10,3.3,1.1$, or $0.37 \mu \mathrm{M})$, RSV $(1 \mu \mathrm{M})$, or DMSO for $24 \mathrm{~h}$. To induce DNA damage, cells were treated with cisplatin $(30 \mu \mathrm{M})$ or DMSO in the presence of test compounds or DMSO for $24 \mathrm{~h}$.
Then whole cell lysates were extracted with radioimmunoprecipitation assay (RIPA) lysis buffer (Beyotime Biotechnology) that contained phenylmethanesulfonyl fluoride (PMSF, Sigma) and protease inhibitor cocktail (Sigma) and subjected to immunoblot assays. Anti-Histone H2A.XS139ph (phospho Ser139) antibodies (GeneTex, GTX127340, 1: 1000 dilution) were used to detect DNA damage levels in cells. Anti- $\beta$ actin antibodies (Proteintech, 60008-1-Ig, 1:1000 dilution) were used to probe $\beta$-actin as a control.

\section{Cellular localization of TyrRS}

HeLa cells were treated with compound 7 (30, 10, 3.3, and 1.1 $\mu \mathrm{M})$, compound $\mathbf{1 1}(30,10,3.3$, and $1.1 \mu \mathrm{M})$, RSV $(1 \mu \mathrm{M})$, or DMSO for $8 \mathrm{~h}$. Cells cultured in serum-free media for $8 \mathrm{~h}$ were under serum starvation. The cytoplasmic and nuclear fractions were separated by NE-PER ${ }^{\circledR}$ nuclear and cytoplasmic extraction reagents (Thermo Scientific, 78835) as per the manufacturer's instructions. Both fractions were then subjected to immunoblot assays. Anti-tyrosyl tRNA synthetase antibodies (Abcam, ab150429, $1: 1000$ dilution) were used to probe TyrRS in the nucleus and cytoplasm. Anti-GAPDH antibodies (Proteintech, 60004-1-Ig, 1 : 1000 dilution) and anti-Lamin B1 (Proteintech, 1298.7-1-AP, $1: 1000$ dilution) antibodies were used to probe GAPDH and Lamin B1 as controls for the cytoplasmic and nuclear fractions, respectively.

\section{Immunoblot assays}

Proteins from cells were resolved by $12 \%$ or $10 \%$ sodium dodecyl sulfate polyacrylamide gel electrophoresis (SDS-PAGE) and transferred to polyvinylidene fluoride (PVDF) membranes (Millipore). The membranes were blocked with TBS-T blocking buffer (5\% nonfat milk in TBS-T) for 1-4 $\mathrm{h}$ at RT and then probed with primary antibodies overnight at $4{ }^{\circ} \mathrm{C}$ with gentle shaking. After three washes with TBS-T, the membranes were incubated with horseradish peroxidase-conjugated goat antimouse or anti-rabbit antibody (Proteintech, $1: 5000$ dilution) for $1 \mathrm{~h}$ at $37^{\circ} \mathrm{C}$ with gentle shaking. After extensive washing with TBS-T, the membranes were preserved in TBS, and the immunoblots were visualized by ECL (Abbkine). Immunoblots were quantified using Adobe photoshop software.

\section{Conclusions}

A structure-based docking strategy was adopted to screen for novel TyrRS inhibitors. After two campaigns including virtual screening and bioassays, hit compounds with the novel scaffold 5,7-dihydroxy-2-phenyl-4H-chromen-4-one were selected for structural optimization and SAR analysis. The most active compound against TyrRS was compound $\mathbf{1 1}$ with a $K_{\mathrm{i}}$ value of $8.8 \mu \mathrm{M}$. The results of orthogonal assays, including DSC and DSF assays, revealed that these compounds bind to the active site of TyrRS. Furthermore, compound $\mathbf{1 1}$ could protect against the DNA damage caused by cisplatin in intact HeLa cells. Therefore, these compounds represent useful probes for further research into the biological function of TyrRS. 


\section{Conflicts of interest}

There are no conflicts to declare.

\section{Acknowledgements}

This work was supported by the National Natural Science Foundation of China (81573349, 81773633, and 21772130), National Science and Technology Major Project (2018ZX09711002-014-002, 2018ZX09711002-011-019, and 2018ZX09711003-003-006), and 1.3.5 project for disciplines of excellence, West China Hospital, Sichuan University.

\section{Notes and references}

1 J. A. Baur, K. J. Pearson and N. L. Price, Resveratrol improves health and survival of mice on a high-calorie diet, Nature, 2006, 444, 337-342.

2 S. J. Park, F. Ahmad and A. Philp, Resveratrol ameliorates aging-related metabolic phenotypes by inhibiting cAMP phosphodiesterases, Cell, 2012, 148, 421-433.

3 M. Jang, L. Cai and G. O. Udeani, Cancer chemopreventive activity of resveratrol, a natural product derived from grapes, Science, 1997, 275, 218-220.

4 A. Riba, L. Deres and B. Sumegi, Cardioprotective Effect of Resveratrol in a Postinfarction Heart Failure Model, Oxid. Med. Cell. Longevity, 2017, 2017, 6819281.

5 D. A. Sinclair and L. Guarente, Small-molecule allosteric activators of sirtuins, Annu. Rev. Pharmacol. Toxicol., 2014, 54, 363-380.

6 M. Viswanathan, S. K. Kim and A. Berdichevsky, A role for SIR-2.1 regulation of ER stress response genes in determining C. elegans life span, Dev. Cell, 2005, 9, 605-615.

$7 \mathrm{M}$. Sajish and P. Schimmel, A human tRNA synthetase is a potent PARP1-activating effector target for resveratrol, Nature, 2015, 519, 370-373.

8 S. S. Kulkarni and C. Cantó, The molecular targets of resveratrol, BBA, Mol. Basis Dis., 2015, 1852, 1114-1123.

9 N. Wei, Y. Shi and L. N. Truong, Oxidative stress diverts tRNA synthetase to nucleus for protection against DNA damage, Mol. Cell, 2014, 56, 323-332.

10 X. Cao, C. Li and S. Xiao, Acetylation promotes TyrRS nuclear translocation to prevent oxidative damage, Proc. Natl. Acad. Sci. U. S. A., 2017, 114, 687-692.

11 X. Luo and W. L. Kraus, On PAR with PARP: cellular stress signaling through poly(ADP-ribose) and PARP-1, Genes Dev., 2012, 26, 417-432.

12 L. Pirola and S. Fröjdö, Resveratrol: One molecule, many targets, IUBMB Life, 2008, 60, 323-332.

13 F. Mao, J. Yan and J. Li, New multi-target-directed small molecules against Alzheimer's disease: a combination of resveratrol and clioquinol, Org. Biomol. Chem., 2014, 12, 5936-5944.

14 R. I. Tennen, E. Michishita-Kioi and K. F. Chua, Finding a target for resveratrol, Cell, 2012, 148, 387-389.

15 M.-H. Lee, B. Y. Choi and J. K. Kundu, Resveratrol suppresses growth of human ovarian cancer cells in culture and in a murine xenograft model: eukaryotic elongation factor $1 \mathrm{~A} 2$ as a potential target, Cancer Res., 2009, 69, 7449-7458.

16 S. H. Yang, Q. Sun and H. Xiong, Discovery of a butyrylcholinesterase-specific probe via a structure-based design strategy, Chem. Commun., 2017, 53, 3952-3955.

17 C. M. Lombardo, I. S. Martinez and S. Haider, Structurebased design of selective high-affinity telomeric quadruplex-binding ligands, Chem. Commun., 2010, 46, 9116-9118.

18 S. Huang, C. Song and X. Wang, Discovery of New SIRT2 Inhibitors by Utilizing a Consensus Docking/Scoring Strategy and Structure-Activity Relationship Analysis, J. Chem. Inf. Model., 2017, 57, 669-679.

19 J. Baell and M. A. Walters, Chemistry: Chemical con artists foil drug discovery, Nature, 2014, 513, 481-483.

20 K. Ding, Y. Lu and Z. Nikolovska-Coleska, Structure-based design of potent non-peptide MDM2 inhibitors, J. Am. Chem. Soc., 2005, 127, 10130-10131.

21 E. Perola, W. P. Walters and P. S. Charifson, A detailed comparison of current docking and scoring methods on systems of pharmaceutical relevance, Proteins, 2004, 56, 235-249.

22 G.-B. Li, L.-L. Yang and W.-J. Wang, ID-Score: a new empirical scoring function based on a comprehensive set of descriptors related to protein-ligand interactions, $\mathrm{J}$. Chem. Inf. Model., 2013, 53, 592-600.

23 H. Park, T. T. Dao and H. P. Kim, Synthesis and inhibition of PGE2 production of 6,8-disubstituted chrysin derivatives, Eur. J. Med. Chem., 2005, 40, 943-948.

24 I. Ryu, H. Matsubara and S. Yasuda, Phase-vanishing reactions that use fluorous media as a phase screen. Facile, controlled bromination of alkenes by dibromine and dealkylation of aromatic ethers by boron tribromide, $J$. Am. Chem. Soc., 2002, 124, 12946-12947.

25 F. H. Niesen, H. Berglund and M. Vedadi, The use of differential scanning fluorimetry to detect ligand interactions that promote protein stability, Nat. Protoc., 2007, 2, 2212-2221.

26 J. V. Rodrigues, V. Prosinecki and I. Marrucho, Protein stability in an ionic liquid milieu: on the use of differential scanning fluorimetry, Phys. Chem. Chem. Phys., 2011, 13, 13614-13616.

27 M. M. Knopp, K. Löbmann and D. P. Elder, Recent advances and potential applications of modulated differential scanning calorimetry (mDSC) in drug development, Eur. J. Pharm. Sci., 2016, 87, 164-173.

28 P. Alam, A. S. Abdelhameed and R. K. Rajpoot, Interplay of multiple interaction forces: Binding of tyrosine kinase inhibitor nintedanib with human serum albumin, $J$. Photochem. Photobiol., B, 2016, 157, 70-76.

29 A. S. Abdelhameed, P. Alam and R. H. Khan, Binding of janus kinase inhibitor tofacitinib with human serum albumin: multi-technique approach, J. Biomol. Struct. Dyn., 2016, 34, 2037-2044.

30 M. Epple, U. Sazama and A. Reller, Simultaneous X-ray absorption fine-structure spectroscopy (XAFS) and 
differential scanning calorimetry (DSC), Chem. Commun., 1996, 1755-1756.

31 R. W. Harkness, S. Slavkovic and P. E. Johnson, Rapid characterization of folding and binding interactions with thermolabile ligands by DSC, Chem. Commun., 2016, 52, 13471-13474.

32 H.-m. Chow and K. Herrup, Genomic integrity and the ageing brain, Nat. Rev. Neurosci., 2015, 16, 672.

33 W. P. Roos, A. D. Thomas and B. Kaina, DNA damage and the balance between survival and death in cancer biology, Nat. Rev. Cancer, 2016, 16, 20.

34 C. A. Lipinski, F. Lombardo and B. W. Dominy, Experimental and computational approaches to estimate solubility and permeability in drug discovery and development settings, Adv. Drug Delivery Rev., 2012, 64, 4-17.

35 D. F. Veber, S. R. Johnson and H.-Y. Cheng, Molecular properties that influence the oral bioavailability of drug candidates, J. Med. Chem., 2002, 45, 2615-2623.

36 X. L. Yang, R. J. Skene and D. E. McRee, Crystal structure of a human aminoacyl-tRNA synthetase cytokine, Proc. Natl. Acad. Sci. U. S. A., 2002, 99, 15369-15374.

37 M. Sajish, Q. Zhou and S. Kishi, Trp-tRNA synthetase bridges DNA-PKcs to PARP-1 to link IFN-gamma and p53 signaling, Nat. Chem. Biol., 2012, 8, 547-554. 